\title{
ASCA and BeppoSAX observations of the peculiar X-ray source 4U1700+24/HD154791
}

\author{
D. Dal Fiume ${ }^{1}$, N. Masetti ${ }^{1}$, C. Bartolini ${ }^{2}$, S. Del Sordo ${ }^{3}$, F. Frontera ${ }^{4}$, A.

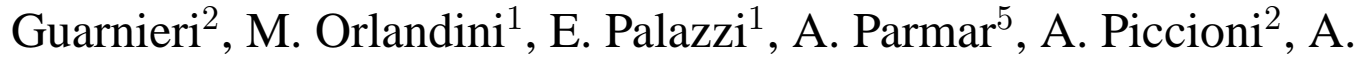 \\ Santangelo ${ }^{3}$, A. Segreto ${ }^{3}$ \\ ${ }^{1}$ Istituto TESRE/CNR, via Gobetti 101, 40129 Bologna, Italy \\ ${ }^{2}$ Dipartimento di Astronomia, Universitá di Bologna, via Ranzani 1, 40127 Bologna, Italy \\ ${ }^{3}$ IFCAI/CNR, via U. La Malfa 153, 90146 Palermo, Italy \\ ${ }^{4}$ Istituto TeSRE and Dipartimento di Fisica, Universitá di Ferrara, via Paradiso 1, 44100 Ferrara, Italy \\ ${ }^{5}$ Space Science Department, ESA, ESTEC, Noordwjik, The Netherlands
}

\begin{abstract}
The X-ray source 4U1700+24/HD154791 is one of the few galactic sources whose counterpart is an evolved $\mathrm{M}$ star [1-3]. In X-rays the source shows extreme erratic variability and a complex and variable spectrum. While this strongly suggests accretion onto a compact object, no clear diagnosis of binarity was done up to now. We report on ASCA and BeppoSAX X-ray broad band observations of this source and on ground optical observations from the Loiano $1.5 \mathrm{~m}$ telescope.
\end{abstract}

\section{INTRODUCTION}

In optical astronomy the identification of a binary system comes in most cases from the observation of photometric and/or radial velocity variations. As not all X-ray binaries have known optical counterparts, a further effective criterium in galactic X-ray astronomy for the identification of a binary system with an accreting compact object was often based on the observed X-ray luminosity. For X-ray binaries harbouring a neutron star or possibly a black hole, luminosities $\mathrm{L}_{X}$ of the order of $10^{34}-10^{35} \mathrm{erg} \mathrm{s}^{-1}$ are easily reached. The diagnosis of the presence of a neutron star in most cases is directly confirmed by the observation of pulsations or thermonuclear bursts, apart from bright persistent Low Mass X-Ray Binaries (LMXRBs). X-ray binaries harbouring white dwarfs also show some distinctive features. As an example in polars and intermediate polars optical and UV observations often reveal the distinctive signatures of the presence of a white dwarf in the system. Orbital periods and light curves also add unambiguous and reliable evidence of the presence of white dwarfs in this class of X-ray binaries.

For a number of X-ray sources the identification of a class or even the diagnosis of binarity is rather difficult, especially when the observed X-ray luminosity is $\leq 10^{33} \mathrm{erg} \mathrm{s}^{-1}$. $4 \mathrm{U} 1700+24 / \mathrm{HD} 154791$ belongs to this class. The optical counterpart was identified by 
Garcia et al. [2] as a late type giant on the basis of the positional coincidence with a HEAO1-A3 error box. The optical spectrum of this giant looks quite normal $[1,2]$, even if Gaudenzi and Polcaro [3] find some interesting and variable features in its spectrum. Variable UV line emission was detected [1,2] in different IUE pointings, showing at last some unusual features in the emission from this otherwise normal giant. These high excitation lines are likely linked to the same mechanism that produces the observed X-ray emission. In spite of various attempts, no evidence of a binary orbit was obtained from radial velocity analysis of optical spectra.

The $\mathrm{X}$-ray source shows extreme erratic variability, but no pulsations were detected. The rapid (10-1000 s) time variability is strongly suggestive of turbulent accretion, often ob-

served in X-ray binaries. The $\mathrm{X}$-ray spectrum is rather energetic and was measured up to $10 \mathrm{keV}$. The $\mathrm{X}$-ray luminosity $\mathrm{L}_{X} \sim 10^{33} \mathrm{erg} \mathrm{s}^{-1}$ at an assumed distance of $730 \mathrm{pc}$ [2] may be marginally consistent with coronal emission, even if an evolved giant is not expected to be a strong X-ray emitter. Therefore the picture emerging from observations gives only hints in favour of a binary system, given that no "classical" feature to be associated to the presence of a compact object was found.

We have observed this source for $\sim 15$ years both with $\mathrm{X}$-ray satellites (EXOSAT, ASCA and BeppoSAX ) and with ground optical observations from the Loiano $1.5 \mathrm{~m}$ and $0.6 \mathrm{~m}$ telescopes of the Bologna Astronomical Observatory. Here we report on the ASCA and BeppoSAX observations, performed respectively on March 8, 1995 and on March 27, 1998. We also report on photometric optical UBVRI monitoring.

\section{OBSERVATIONS}

In Figure 1 we show the observed 1.5-9 keV count rate from the GIS2 and GIS3 instruments on board ASCA and the 1.5-10 keV observed count rate from the MECS2 and MECS3 instruments on board BeppoSAX. A clear increase of the 4U1700+24 count rate was detected in November 1997 by RXTE/ASM (http://space.mit.edu/XTE/ASM_lc.html ). The BeppoSAX observation was performed approximately five months after this event, when the source had already recovered its quiescent flux. The substantial erratic variability already detected with EXOSAT [1] is clearly present also in both observations. The source flux in the BeppoSAX observation is significantly lower than that in the ASCA observation. The erratic source variability is clearly visible in the Power Spectral Density (PSD) shown in Figure 2, calculated on the time series of GIS2 and GIS3 count rate binned on $0.1 \mathrm{~s}$. The spectra were calculated for runs with typical duration of 3000s. The PSD shown in Figure 2 is obtained by averaging the spectra of different runs and by summing adjacent frequencies with a logarithmic rebinning. The observed $\mathrm{X}$-ray source luminosity $(2-10 \mathrm{keV})$ was $L_{X}=1.7 \times 10^{33} \mathrm{erg} \mathrm{s}^{-1}$ in the ASCA observation and $L_{X}=6 \times 10^{32} \mathrm{erg} \mathrm{s}^{-1}$ in the BeppoSAX observation assuming a distance of $\sim 700 \mathrm{pc}[2]$.

The X-ray energy spectrum cannot be fitted by simple single component models. The high energy ( $>2 \mathrm{keV}$ ) spectrum can be fitted by an absorbed thermal continuum, but the extrapolation of such a model at lower energies lies significantly below the measured 

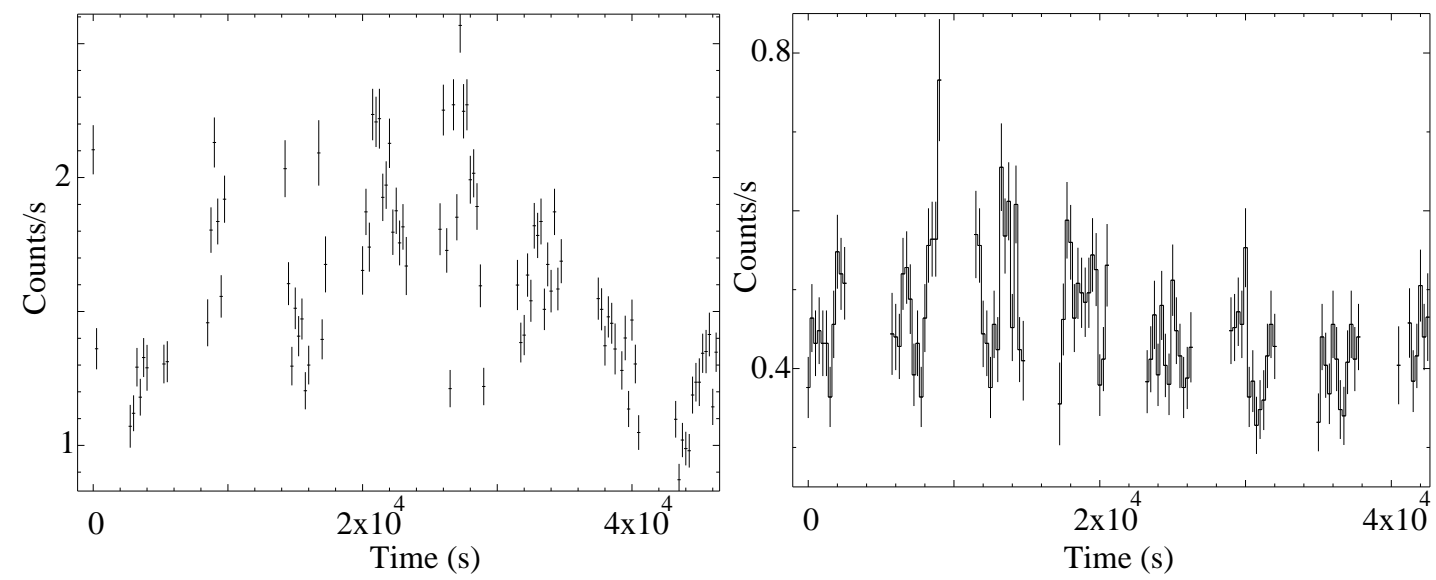

FIGURE 1. Count rate time series from the ASCA and BeppoSAX observations

spectrum, both in ASCA and in BeppoSAX observations.

For a thermal model, similar to that used in the low luminosity source $\gamma$ Cas (a suspected Be/white dwarf binary $[4,5])$, the addition of a complex absorber (e.g. a partial absorber) is needed to model the low energy part of the spectrum. The lack of Fe emission line however requires a very low $\mathrm{Fe}$ abundance.

As an example the count rate spectra from the ASCA and BeppoSAX observations fitted with an optically thin thermal bremsstrahlung continuum with partial absorber ("bremss" and "pcfabs" models in XSPEC) are shown in Figure 3. The BeppoSAX spectrum is softer than that observed with ASCA, and very similar to that observed with EXOSAT [1] at almost exactly the same flux level of the BeppoSAX observation. Optical observations were performed at the Loiano $1.5 \mathrm{~m}$ telescope of the Bologna Astronomical Observatory during the last 15 years. HD154791, the optical counterpart of the $\mathrm{X}$-ray source, is a M2-M3 giant [2,3] with a rather normal optical spectrum. A simple comparison with M1-M3 III templates shows a close match with the M2 template of HD104216. In Figure 4 we report the long term UBVRI photometry of HD154791. No clear long term trend is visible. Some variability is present, in particular in the $\mathrm{U}$ measurements, that may be intrinsic to the source. The long-term spectral/photometric monitoring of the source is continuing.

\section{DISCUSSION}

The observations we report still cannot be used to perform a "classical" diagnosis of binarity. We nevertheless note some interesting similarities with other low luminosity $\mathrm{X}$-ray sources. In particular some interesting similarities can be found with the $\mathrm{X}$-ray emission from $\gamma$ Cas. The power spectrum is strikingly similar and the energy spectrum shows a similar shape, even if no iron line is detected in 4U1700+24 .

However this close resemblance of the properties of the X-ray emission does not help to determine the presence of a compact object in a binary system, as for $\gamma$ Cas itself

the diagnosis of binarity is not completely assessed. In fact Owens et al. [5] favour the 


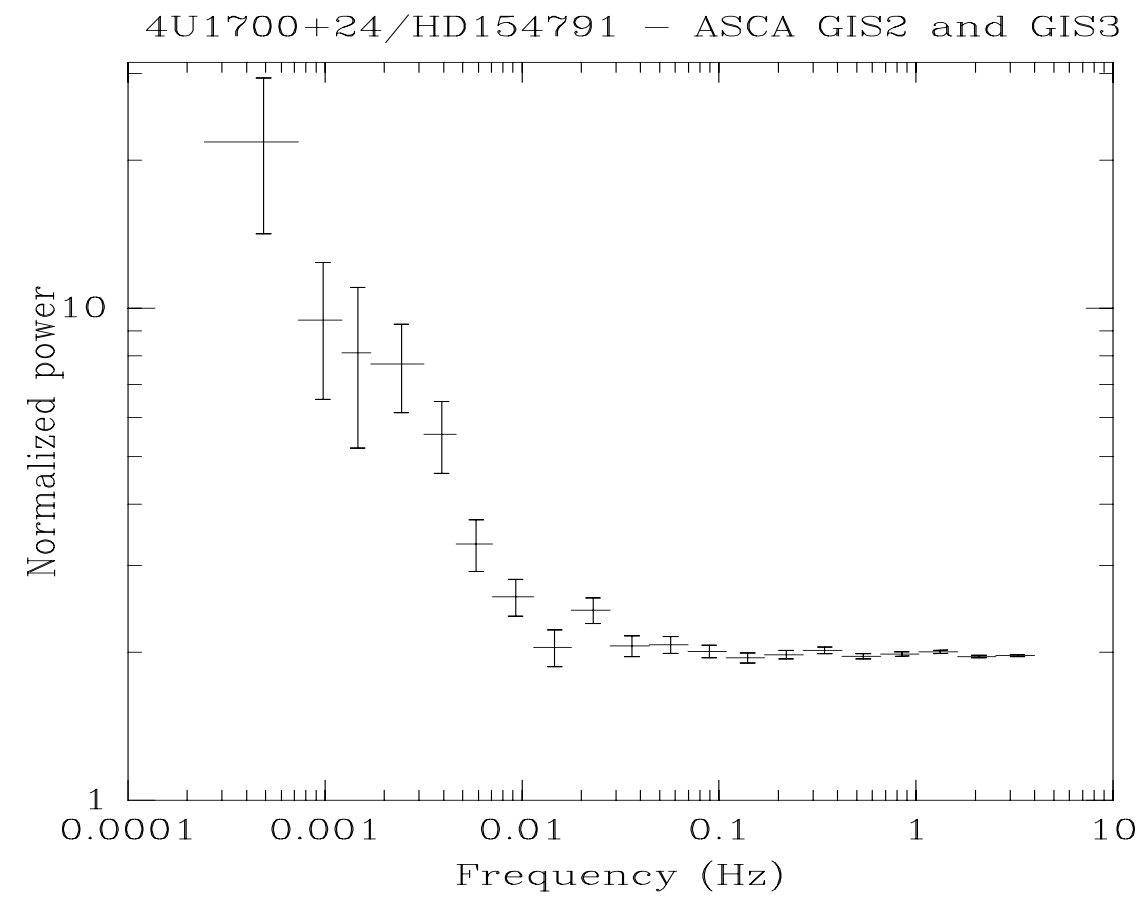

FIGURE 2. Power Spectral Density from ASCA observation
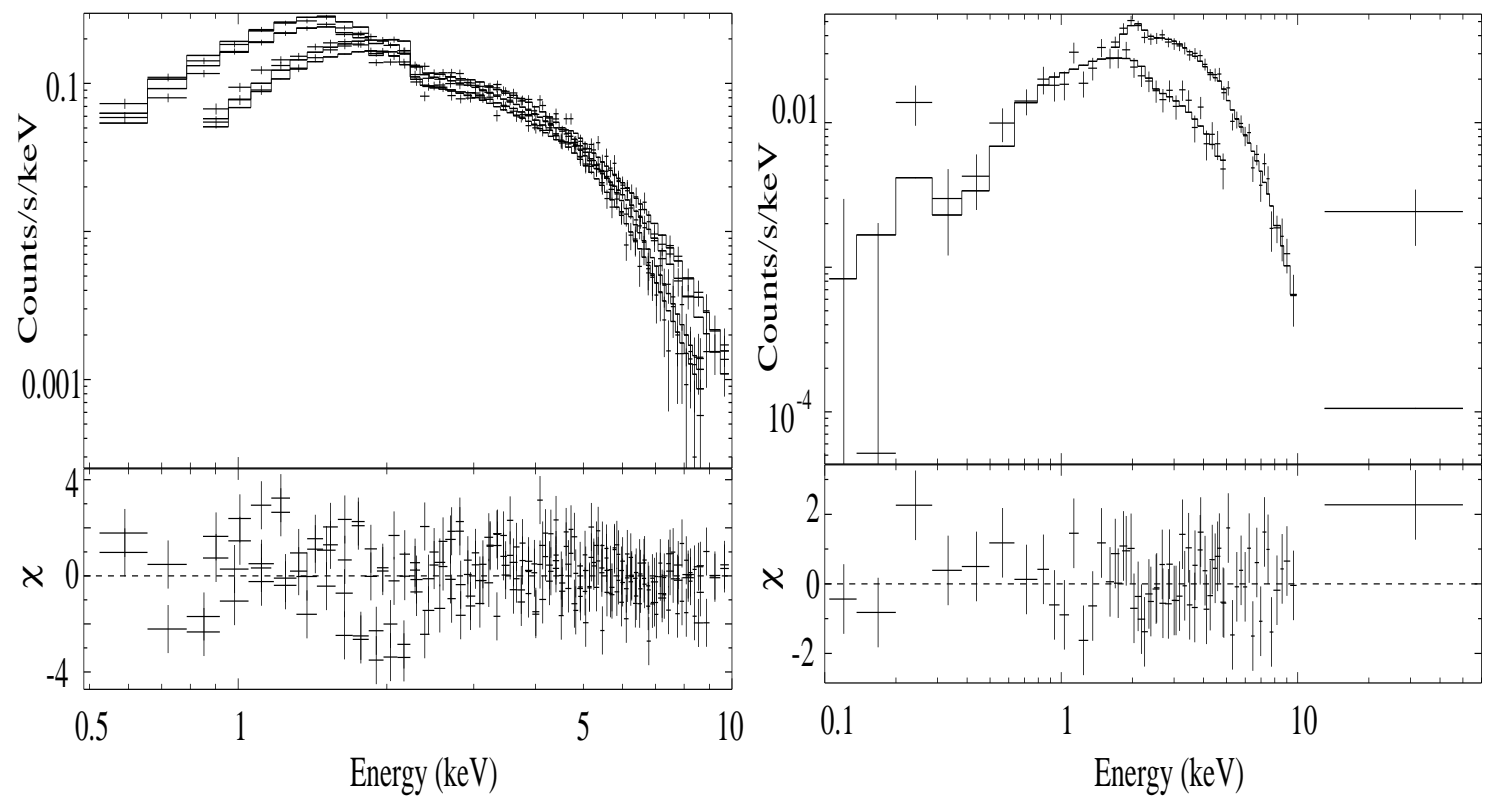

FIGURE 3. Left: fit to ASCA data. Partial covering fraction: $0.75 \pm 0.01$. Temperature $k T=6.25 \pm 0.2$. Reduced $\chi_{d o f}^{2}$ : 1.55 Right: fit to BeppoSAX data. Partial covering fraction: $0.72 \pm 0.04$. Temperature $\mathrm{kT}=3.6 \pm 0.3$. Reduced $\chi_{\text {dof }}^{2}: 0.9$ 


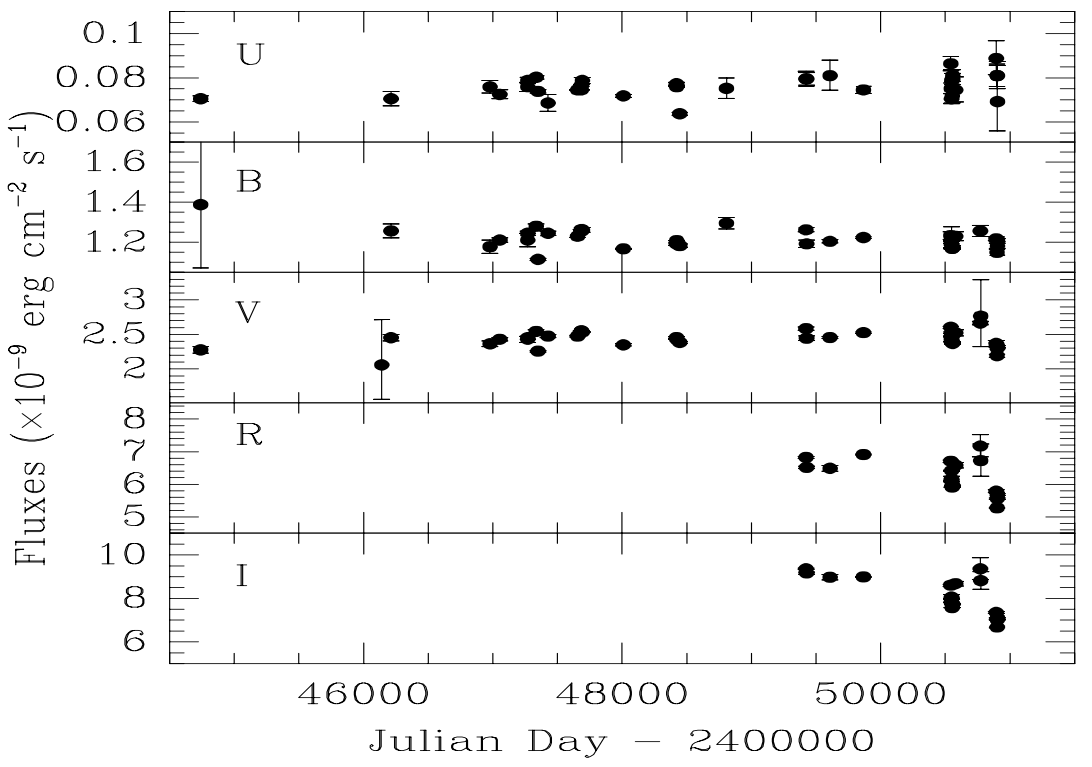

FIGURE 4. UBVRI long term variability of HD154791

hypothesis of a WD binary, but a completely different point of view is based on recent UV/X-ray observations of $\gamma$ Cas [6]. Smith et al. support the hypothesis that the Xray emission of $\gamma$ Cas comes from continuous flaring from the Be star. This hypothesis cannot be easily adapted to the case of 4U1700+24/HD154791, as the much colder M giant star should not be expected to have strong and persistent $\mathrm{X}$-ray flaring activity. If this is the case, i.e. the observed X-ray emission from 4U1700+24 is coronal, HD154791 should be an exception in its own class. If the similarity of the properties of the X-ray emission from $4 \mathrm{U} 1700+24$ and $\gamma$ Cas comes from a common origin, we suggest that the WD binary hypothesis is much more comfortable and more easily met.

Acknowledgements. This research is supported by the Agenzia Spaziale Italiana (ASI) and the Consiglio Nazionale delle Ricerche (CNR) of Italy. BeppoSAX is a joint program of ASI and of the Netherlands Agency for Aerospace Programs (NIVR). The ASCA observation was performed as part of the joint ESA/Japan scientific program. CB, AG and AP acknowledge a grant from "Progetti di ricerca ex-quota 60\%" of Bologna University.

\section{REFERENCES}

1. Dal Fiume, D. et al. 1990 Il Nuovo Cimento C, 13, 481

2. Garcia, M. et al. 1983, ApJ, 267, 291

3. Gaudenzi, S. F., Polcaro, V. F. 1999 Astron. Astrophys., 347, 473

4. Kubo, S. et al. 1998 PASJ, 50, 417

5. Owens, A. et al. 1999 Astron. Astrophys, 348, 170

6. Smith, M. A. et al. $1998 A p J, \mathbf{5 0 3}, 877$. 